\title{
Niveles de Resiliencia en Jóvenes Deportistas de la Federación Deportiva de Manabí
}

\section{Resilience levels in young athletes of the Manabí sports federation.}

\author{
Anicia Tarazona Meza, MSc ${ }^{(1)}$ \\ Inger Maitta Rosado, MSc (2) \\ Joselyn Cevallos Alcívar (3)
}

(1) Universidad Técnica de Manabí, Facultad de Ciencias Humanísticas y Sociales, Magister en Gerencia Educativa, Manabí-Ecuador, atarazona@utm.edu.ec

(2) Universidad Técnica de Manabí, Facultad de Ciencias Humanísticas y Sociales, Magister en Gerencia

Educativa, Manabí-Ecuador, imaitta@utm.edu.ec

(3) Egresada de la carrera de Psicología Clínica, jcevallos0330@utm.edu.ec

Contacto: jcevallos0330@gmail.com

Recibido: 18-02-2020 Aprobado: 23-03-2020

\section{Resumen:}

El objetivo de la investigación es comparar los niveles de resiliencia por deportes en jóvenes deportistas de la Federación Deportiva de Manabí, con el uso de una metodología con aspectos científicos basados en el proceso inductivo-deductivo y el método de campo. Con técnicas e instrumentos que validan la aplicación del Test SV-RES, Registro de Observación, Cuadro de entrada y salida (deporte, edad, cantidad, nivel de resiliencia y matriz de resiliencia). Población: Deportistas entre los 18 a 25 años en los 3 deportes que fueron, Escalada, Ajedrez y Boxeo. Los resultados abordaron una dimensión inmediata en donde opera la resiliencia de forma adecuada a los deportistas motivo de investigación, los cuales dependen de las capacidades condicionales: velocidad, fuerza, resistencia y flexibilidad para evaluar el nivel de resiliencia personal atreves del control único de la muestra, donde el mismo funciona de manera objetiva con la concentración mental, ante pérdidas, golpes y estados sistemáticos a los que someten al competidor, en el caso de los atletas ajedrecistas, su nivel de resiliencia varía a causa de exigencia mental y la confianza, y las tres escalas medidas que fueron ( Yo soy- Yo estoy, Yo tengo. Yo puedo), operan de manera adecuada en cada uno. Permitiendo establecer una ventana abierta en el proceso de preparación de estos deportes, a través de la presencia del profesional en psicología de forma contínua para elevar la autoestima de los mismos.

Palabras clave:

Psicología deportiva, Resiliencia deportiva, Rendimiento deportivo, Estados de resiliencia

\begin{abstract}
:
The objective of the research is to compare the levels of sports resilience in young athletes of the Sports Federation of Manabí, with the use of a methodology with scientific aspects based on the inductive-deductive process and the field method. With techniques and instruments that validate the application of the SV-RES Test, Observation Record, Entry and Exit Chart (sport, age, quantity, level of resilience and resilience matrix). Population: Athletes between 18 and 25 years in the 3 sports that were, Climbing, Chess and Boxing. The results addressed an immediate dimension where resilience operates adequately for the athletes under investigation, which depend on the conditional capacities: speed, strength, endurance and flexibility to assess the level of personal resilience through the unique control of the sample., where it works objectively with mental concentration, in the face of losses, blows and systematic states to which the competitor is subjected, in the case of chess athletes, their level of resilience varies due to mental demand and confidence, and the three measured scales that were (I am, I have. I can), operate properly in each one. Allowing to establish an open window in the process of preparing these sports, through the presence of the professional in psychology continuously to raise their self-esteem.
\end{abstract}

Keywords:

Sports psychology, Sports resilience, Sports performance, Resilience states 


\section{Introducción:}

El fenómeno que representa la resiliencia no es nuevo, aunque lo parezca, su historia marcha junto a la tradición del hombre y se fundamenta en la creencia de que lo positivo siempre tiene que ser superior a lo negativo y que las fortalezas tendrán un mayor peso que lo que las dificultades pueden representar. (Tarazona, A, p. 11).

La vida profesional de los deportistas está llena de fluctuaciones, de victorias y derrotas que pueden influir negativamente en su funcionamiento psicológico. La necesidad de una mejor comprensión de las habilidades potenciales de los deportistas para afrontar experiencias negativas, haciéndoles menos vulnerables a los efectos de acontecimientos estresantes, ha conducido a los especialistas al examen en profundidad de variables moderadoras que faciliten el desarrollo de respuestas positivas a situaciones de estrés y adversidad, reforzando las capacidades de afrontamiento de las mismas. (García, 2014, p.83)

Según Chacón (2016). "El estudio de la resiliencia como capacidad para superar situaciones de adversidad ha tomado protagonismo en la última década, especialmente en la rama de la psicología deportiva".

El deporte y la actividad física fomentan ciertos aspectos personales muy relacionados con la salud y, con ello, el aumento de los niveles de Resiliencia. Así pues, este estudio tiene como objetivo comprobar si los sujetos que practican deporte presentan diferencias en cuanto a los niveles de resiliencia respecto a los que no lo practican; entre los que compiten y los que no; y si existe una relación entre las horas semanales de actividad física realizadas por los sujetos y sus niveles de Resiliencia. (Peris, 2017, p. 1).

En el desarrollo de la investigación fue importante determinar los métodos y técnicas que fueron aplicados para realización de este proceso, en primer aspecto la observación científica fue uno de las principales herramientas utilizadas para procesar el trabajo de campo. Según Campos (2012).La observación es la forma más sistematizada y lógica para el registro visual y verificable de lo que se pretende conocer; es decir, es captar de la manera más objetiva posible, lo que ocurre en el mundo real, ya sea para describirlo, analizarlo o explicarlo desde una perspectiva científica. El proceso inductivo-deductivo, destacó la actividad mediante el proceso del desarrollo de campo, proponiendo nuevos procesos de manera asertiva y eliminando la redundancia de forma crítica aplicativa, para esto se procesó a realizar una triangulación que constó del: análisis-síntesis, comparativo, bibliográfico, teniendo en cuenta además el método correlacionar y de campo. Con resultados que abordaron una dimensión inmediata donde opera la resiliencia de forma adecuada en los deportistas, en la disciplinas que exigen fuerza, y destreza física como lo son: escalada y boxeo el nivel de resiliencia personal es un poco elevado porque funciona de manera objetiva con la concentración mental, ante pérdidas, golpes y estados sistemáticos a los que someten al competidor, en el caso de los deportistas del área de ajedrez su nivel de resiliencia varía a causa de exigencia mental y la confianza, y las tres escalas medidas que fueron ( Yo soy- Yo estoy, Yo tengo. Yo puedo), operan de manera adecuada en cada uno. La presencia del profesional en Psicología debe de ser de forma continua y metodológica para procesar el trabajo que se realiza durante la consultoría y exteriorización con lo sucedido con el deportista. El presente trabajo tuvo como objetivo comparar los niveles de resiliencia por categorías deportivas en los jóvenes deportistas de la Federación Deportiva de Manabí.

\section{Materiales y métodos}

En el desarrollo de la investigación fue importante determinar los métodos y técnicas que se aplicaron para realizar la contribución expuesta: Primer momento se enfoca en la Federación Deportiva de Manabí, en un periodo comprendido de 3 meses. Como segundo momento el aspecto la observación científica, fue una de las principales herramientas utilizadas para procesar el trabajo de campo. El segundo momento también comprende, el proceso inductivo-deductivo, del cual se procesó a realizar una triangulación que constó del: análisis-síntesis, comparativo, bibliográfico, teniendo en cuenta además el método de campo. Instrumentos: Test SV-RES (Saavedra \& Villalta, 2008)que está constituido por 60 ítems, dividido en 12 dimensiones específicas de la resiliencia, inspirado en el modelo de verbalizaciones (yo soy- yo estoy, yo tengo, yo puedo) de Grotberg y Saavedra. Aplicando el test de resiliencia SV-RES a un grupo de 40 deportistas. Además, el registro de la observación y el cuadro de entrada y salida (deporte, edad, nivel de resiliencia y matríz de resiliencia). Población: Deportistas entre los 18 a 25 años. Muestra: Tipo de muestreo no probabilístico, se utilizarán un muestreo discrecional, considerando el rango de edad de 18 a 25 años, por categoría de los deportes que se practican en la Federación, excluyendo los deportistas menores de 17 años. Adicional se trabajó con las categorías deportivas que son: Ajedrez, Boxeo y Escalada, los cuales fueron medidos en los niveles de referencias, y los tipos de frecuencia por cada nivel, basado en las tablas de aplicación del desarrollo y aporte de resultados de la investigación. 


\section{Resultados}

Para procesar la información obtenida mediante la aplicación del instrumento "Escala de Resiliencia SVRES60 el cual detalla el nivel de resiliencia estructurada en tres campos que son: YO SOY- YO ESTOY- YO TENGO- YO PUEDO. El mecanismo de interpretación de la muestra tomada que obedece a 40 deportistas será validado mediante los factores de la resiliencia, que se adjuntan a un cuadro de indicadores.
En la tabla 1, se puede observar los puntajes percentiles, que fueron expresados en cada uno de los niveles de resilencia, los cuales fueron adoptados de acuerdo al modelo del Test SV-RES60.

\begin{tabular}{|c|c|c|}
\hline Puntaje percentil & $0-25$ & Bajo \\
\hline Puntaje percentil & $26-74$ & Promedio \\
\hline Puntaje percentil & $75-99$ & Alto \\
\hline
\end{tabular}

Tabla 1.- Interpretación del puntaje percentil Fuente: Descripción de la interpretación del puntaje percentil

\section{NIVEL DE RESILENCIA DE LA MUESTRA TOTAL}

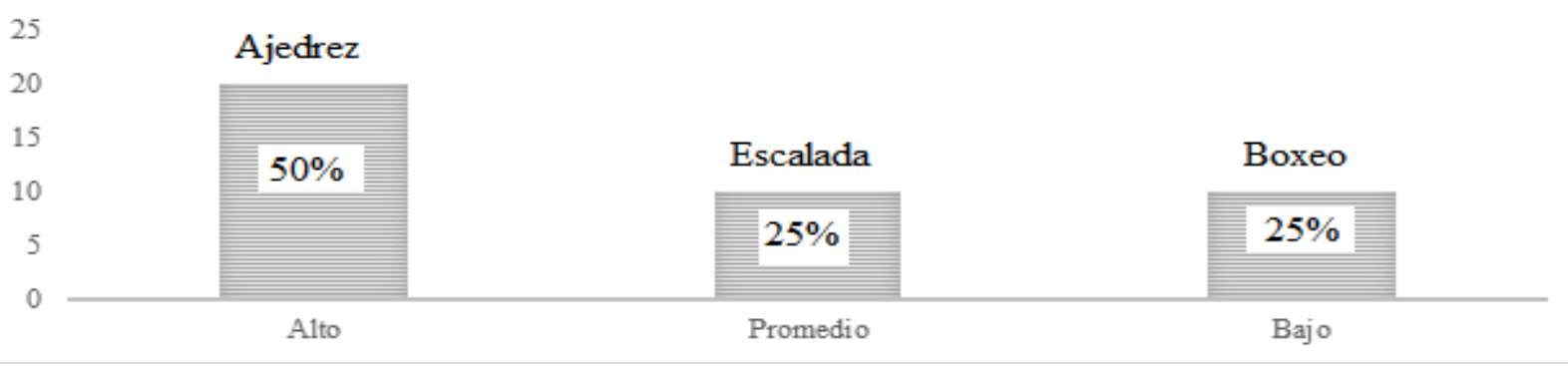

Figura 1. Nivel de Resilencia en categorías deportivas.

Fuente: Jóvenes Deportistas de la Federación Deportiva de Manabí.

De acuerdo con el grafico 1, que ha sido elaborado con el teste SV-RES60, evaluado el nivel de resiliencia aplicado a 40 deportistas de la Federación Deportiva de Manabí. Se puede determinar que el $50 \%$ según la escala estadística equivalente a 20 personas responden que su nivel de resiliencia es elevado, mientras que un $25 \%$ según la escala estadística equivalente a 10 persona responden mediante las encuesta que el nivel de resiliencia es promedio, (esto indica que su porcentaje de resiliencia puede mejorar de manejar mayormente asertiva y con guías profesionales) mientras que el siguiente $25 \%$ según la escala estadística, equivalente a 10 personas, su nivel de resiliencia es un poco bajo ( Se puede intervenir de manera objetiva, porque las disciplinas deportivas exigen niveles de resistencia por la carga de competencia dentro de sus estados personales).

\begin{tabular}{|l|l|l|l|l|}
\hline Edad & Deporte & Cantidad & $\begin{array}{l}\text { Nivel de } \\
\text { resilencia }\end{array}$ & $\begin{array}{l}\text { Matriz de } \\
\text { resiliencia }\end{array}$ \\
\hline $18-20$ & Ajedrez & 20 & Alto & Yo tengo \\
\hline $18-22$ & Boxeo & 10 & Promedio & Yo soy, yo estoy \\
\hline $28-25$ & Escalada & 10 & Bajo & Yo puedo \\
\hline
\end{tabular}

Tabla 2.- Análisis de los Resultados de los Niveles de Resiliencia por: edad, deporte, cantidad, nivel de resiliencia y matriz de resiliencia.

Fuente: Descripción de la interpretación del puntaje percentil. 
En la tabla 2 se aprecia que el área deportiva con el mayor número de apropiación del dominio yo tengo, es la ajedrez, esto puede ser producido por el nivel de exigencia mental que tienen los competidores y el estrés mental puede ser un factor el cual se transforma en apropiación del proceso resilente, las edades son cruciales y se puede observar que la gran parte constan de 18 a 22 años, las cuales son propicias para el manejo conductista del ser humano para el enfrentamiento de problemas futuros.

\begin{tabular}{|c|c|c|c|c|}
\hline & $\begin{array}{c}\text { Condiciones de } \\
\text { base }\end{array}$ & Visión de sí mismo & $\begin{array}{c}\text { Visión del } \\
\text { problema }\end{array}$ & Respuestas resilentes \\
\hline Yo soy- yo estoy & F1. Identidad & F2. Autonomía & F3 Satisfacción & F4. Pragmatismo \\
\hline Yo tengo & F5. Vínculos & F6. Redes & F7. Modelo & F8. Metas \\
\hline Yo puedo & F9 Afectividad & F10. Autoeficacia & F11. Aprendizaje & F12 Generatividad \\
\hline
\end{tabular}

Tabla 3.- Análisis de los resultados dividido en los factores del test Sv-Res60

Fuente: Descripción especifica de los 12 factores de la resiliencia del test SV-RES60

La tabla 3, expone los aspectos que están en las tres categorías generales la resilencia, que son Yo soy- yo estoy, el Yo tengo y el Yo puedo, luego podemos observar las diferentes frecuencias que están divididas en los niveles resilentes, ahora los porcentajes y establecen en la tabla 1.

\begin{tabular}{|l|c|}
\hline F1.- Identidad & 15 \\
\hline F2. Autonomía & 10 \\
\hline F3. Satisfacción & 15 \\
\hline F4. Pragmatismo & \\
\hline
\end{tabular}

Tabla 4.- Factor de Resiliencia (Yo soy - yo estoy) Fuente: Descripción del factor de Resiliencia (Yo soy, -yo estoy) del test SV-RES60.

La tabla 4 destaca que 15 de los deportistas respondieron que reconocen su identidad, 10 de los deportistas dijeron tener una autonomía bien identificada y 15 de ellos indican que cumplen una satisfacción en la actividad que realizan. 


\begin{tabular}{|c|c|c|c|c|c|}
\hline Yo soy-yo estoy & $\begin{array}{l}\text { Muy de } \\
\text { acuerdo }\end{array}$ & $\begin{array}{l}\text { De } \\
\text { acuerdo }\end{array}$ & $\begin{array}{l}\text { Ni de acuerdo ni } \\
\text { desacuerdo }\end{array}$ & \begin{tabular}{|l|} 
En \\
desacuerdo
\end{tabular} & $\begin{array}{l}\text { Muy en } \\
\text { desacuerdo }\end{array}$ \\
\hline Una persona con esperanza & 15 & 20 & 5 & & \\
\hline Una persona con buena autoestima & 10 & 25 & 2 & 3 & \\
\hline Optimista respecto al futuro & 20 & 10 & 5 & 4 & 1 \\
\hline Seguro de mis creencias o principios & 10 & 20 & 5 & 5 & \\
\hline Creciendo como persona & 10 & 12 & 15 & 3 & \\
\hline $\begin{array}{l}\text { Rodeado de personas que en general me } \\
\text { ayuden en situaciones difíciles }\end{array}$ & 12 & 30 & 4 & 4 & \\
\hline $\begin{array}{l}\text { En contactos con personas que me } \\
\text { aprecian }\end{array}$ & 15 & 20 & 4 & 1 & \\
\hline Seguro de sí mismo & 10 & 20 & & & 10 \\
\hline Seguro de mis proyectos y metas & 20 & 15 & 3 & 2 & \\
\hline Seguro en el ambiente que vivo & 5 & 15 & 10 & 9 & 1 \\
\hline $\begin{array}{l}\text { Una persona que ha aprendido a salir } \\
\text { adelante en la vida }\end{array}$ & 15 & 15 & 10 & & \\
\hline Un modelo positivo para otras personas & 20 & 10 & 10 & & \\
\hline $\begin{array}{l}\text { Bien integrado en mi lugar de trabajo o } \\
\text { estudio }\end{array}$ & 15 & 25 & & & \\
\hline $\begin{array}{l}\text { Satisfecho con mis relaciones de } \\
\text { amistad }\end{array}$ & 12 & 16 & & & \\
\hline Satisfecho con mis relaciones afectivas & 20 & 20 & & & \\
\hline Una persona práctica & 10 & 25 & 5 & & \\
\hline Una persona con metas en la vida & 10 & 12 & 18 & & \\
\hline Activo frente a mis problemas & 18 & 10 & 12 & & \\
\hline $\begin{array}{l}\text { Revisando constantemente el sentido de } \\
\text { mi vida }\end{array}$ & 15 & 14 & 1 & 10 & \\
\hline Generando soluciones a mis problemas & 15 & 15 & 5 & 5 & \\
\hline
\end{tabular}

Tabla 5.- Factor de Resiliencia (Yo soy - yo estoy).

Fuente: Descripción de los factores de Resiliencia (Yo soy-Yo estoy) del test SV-RES60. 
En este aspecto de análisis fue importante destacar el nivel de resiliencia, para detectar el ajuste de las disciplinas observadas en la Federación Deportiva de Manabí, se seleccionaron 2 ítems de investigación para hacer un proceso de apreciación más indicativo. (Tabla 5)

Factor 8: Seguro de sí mismo (20 de la muestra intervenida) este estado de la persona, revela que la seguridad está ligada al estado de la actividad física lo cual vincula de forma consiste la capacidad de desarrollarse de forma positiva. Según Vidarte (2011), $\mathrm{La}$ actividad física es una herramienta eficaz y efectiva en la promoción de la salud siempre y cuando se incluyan aspectos inherentes al ser humano y no solamente la optimización del espacio físico como elemento primordial de las estrategias de promoción de la salud; de igual forma, se hace necesario el empoderamiento de la comunidad de espacios y políticas que contemplan su manejo y conocimiento, así como la educación de la comunidad, que debe estar encaminada hacia el beneficio individual y colectivo a través de la concientización de la disminución de los factores de riesgo que posibilitan la adquisición de patologías que traerán a largo plazo deterioro de su calidad de vida. (p. 218)

Factor 18: Activo frente a mis problemas (18 de la muestra intervenida), este factor es importante correlacionar los sistemas de enfrentar los problemas de forma adecuada. Ante esta situación, los jóvenes deben dejar de verse como objetos de tratamiento o intervención, y asumirlos como actores y participantes que deben poder actuar y decidir antes las situaciones que afectan y restringen su bienestar y desarrollo. (Parra, 2011, p.16).

Los resultados indican de manera externa que el nivel de resiliencia en los deportistas de la Federación, se encuentra en un nivel adaptado al rango "Muy de acuerdo", lo que significa que el entorno y donde se encuentran desarrollando ha sido de formación personal ante su propio contexto interpersonal.

\begin{tabular}{|l|c|}
\hline F2.vínculos & 15 \\
\hline F6. Redes & 15 \\
\hline F7. Modelo & \\
\hline F8. Metas & 10 \\
\hline
\end{tabular}

Tabla 6.- Factor de Resiliencia (Yo tengo)

Fuente: Descripción del factor de Resiliencia (Yo tengo) del test SV-RES60.

Analizándo la tabla 6 , se puede notar que, de la población intervenida, 15 de los deportistas respondieron de forma continua la frecuencia vínculos, 15 más de ellos respondieron redes y 10 afirmaron la frecuencia metas.

Analizándo la tabla 7, es impotante mencionar que, resultó importante destacar el nivel de resiliencia, para detectar el ajuste de las disciplinas observadas en la Federación Deportiva de Manabí, se seleccionaron 3 ítems de investigación para hacer un proceso de apreciación más indicativo. 


\begin{tabular}{|c|c|c|c|c|c|}
\hline Yo tengo & $\begin{array}{l}\text { Muy de } \\
\text { acuerdo }\end{array}$ & $\begin{array}{c}\text { De } \\
\text { acuerdo }\end{array}$ & $\begin{array}{l}\text { Ni de acuerdo ni } \\
\text { desacuerdo }\end{array}$ & $\begin{array}{c}\text { En } \\
\text { desacuerdo }\end{array}$ & $\begin{array}{l}\text { Muy en } \\
\text { desacuerdo }\end{array}$ \\
\hline Relaciones personales confiables & 20 & 20 & & & \\
\hline Una familia bien estructurada & 25 & 10 & 5 & & \\
\hline Relaciones afectivas sólidas & 20 & 20 & & & \\
\hline Fortaleza interior & 15 & 5 & 10 & & \\
\hline Una vida con sentido & 25 & 15 & & & \\
\hline Acceso a servicios Sociales-públicos & 10 & 20 & 5 & 5 & \\
\hline Personas que me apoyan & 20 & 20 & & & \\
\hline $\begin{array}{l}\text { A quién recurrir en caso de } \\
\text { problemas }\end{array}$ & 10 & 20 & 10 & & \\
\hline $\begin{array}{l}\text { Personas que estimulan mi } \\
\text { autonomía e iniciativa }\end{array}$ & 15 & 10 & 5 & 5 & 5 \\
\hline $\begin{array}{l}\text { Personas que me han orientado y } \\
\text { aconsejado }\end{array}$ & 12 & 12 & 1 & 10 & 5 \\
\hline $\begin{array}{l}\text { Personas que me ayudan a evitar } \\
\text { peligros o problemas }\end{array}$ & 10 & 10 & 10 & 8 & 2 \\
\hline Personas en las cuales puedo confiar & 20 & 10 & 10 & & \\
\hline $\begin{array}{l}\text { Personas que han confiado sus } \\
\text { problemas en mi }\end{array}$ & 35 & 5 & & & \\
\hline $\begin{array}{l}\text { Personas que me ha acompañado } \\
\text { cuando he tenido problemas }\end{array}$ & 20 & 5 & 10 & 5 & \\
\hline Metas a corto plazo & 15 & 15 & & 10 & \\
\hline Mis objetivos claros & 15 & 15 & 5 & 5 & \\
\hline $\begin{array}{l}\text { Personas con quien enfrentar los } \\
\text { problemas }\end{array}$ & 22 & 18 & & & \\
\hline Proyectos a futuro & 15 & 5 & 20 & & \\
\hline Problemas que puedo solucionar & 20 & 5 & 5 & 10 & \\
\hline
\end{tabular}

Tabla 7.- Factor de Resiliencia (Yo tengo)

Fuente: Descripción de los factores de Resiliencia (Yo tengo) del test SV-RES60. 
Factor 22: Una familia bien estructurada ( 25 de la muestra investigada), esto demuestra que la mayoría de los competidores vienen de familias con núcleos sólidos, lo cuales es importante para el desarrollo de ellos mismos. La familia como grupo social, ha cambiado en cuanto a su estructura, formas y modelos, ha incorporado nuevas costumbres como consecuencia de la dinámica transferencia social propia de la globalización, en este artículo, se pretende conducir al Derecho y a las disciplinas en general, hacia una reflexión amplia e incluyente de muchos aspectos que modifican el concepto de esta institución en tiempos de posmodernidad. (Oliva, 2013, p.11), esto se lo puede contrastar con el proceso de esta categoría.

Factor 29: Personas que estimulan mi autonomía e iniciativa (15 de la muestra investigada) Los efectos significativos del apoyo a la autonomía perciba en el óptimo proceso de los deportistas, eso ayuda a los niveles de satisfacción con el estilo del liderazgo. Esto lo afirma Álvarez, 2013. Acotación de este proceso que se lleva a cabo la motivación es uno de los principales factores de apoyo en los deportistas, los cuales estimulan la resiliencia entre los deportistas.

Factor 40: Problemas que puedo solucionar (20 de la muestra investigada) En primera instancia se reconoce que la resolución de problemas es una estrategia metodológica que fomenta un aprendizaje significativo, Además, promueve el desarrollo de habilidades, destrezas y diversas competencias, esto porque se enfrentan a un problema que les plantea una serie de retos y dificultades; sin embargo, al resolverlo, con la ayuda del docente y el empleo de sus habilidades y conocimientos previos, logran asimilar nuevas habilidades y competencias.

\begin{tabular}{|l|l|}
\hline F9.Afectividad & 10 \\
\hline F10. Autoeficacia & 20 \\
\hline F11. Aprendizaje & 5 \\
\hline F12. Generatividad & 5 \\
\hline
\end{tabular}

Tabla 8.- Factor de Resiliencia (Yo puedo)

Fuente: Descripción del factor de Resiliencia (Yo puedo) del test SV-RES60.

Analizando la tabla 8, es necesario mencionar el nivel de resiliencia, para detectar el ajuste de las disciplinas observadas en la Federación Deportiva de Manabí, se seleccionaron 4 ítems de investigación para hacer un proceso de apreciación más indicativo. 10 de ellos respondieron al factor de efectividad, mientras 20 afirman contener un auto eficiencia. Demostrando que se basa en ejes de especificidad dentro de la categoría deportiva a la que pertenecen.

Factor 41: 35 de la muestra investigada se mostraron resilentes al hablar sobre sus emociones)

Factor 44: 40 de la muestra investigada, superan las dificultades que se les presentan en la vida.

Factor 60: 40 de la muestra investigada se proyectan a futuro. 


\begin{tabular}{|c|c|c|c|c|c|}
\hline Yo puedo & $\begin{array}{l}\text { Muy de } \\
\text { acuerdo }\end{array}$ & $\begin{array}{l}\text { De } \\
\text { acuerdo }\end{array}$ & $\begin{array}{l}\text { Ni de acuerdo ni } \\
\text { desacuerdo }\end{array}$ & \begin{tabular}{|l|} 
En \\
Desacuerdo \\
\end{tabular} & $\begin{array}{l}\text { Muy en } \\
\text { desacuerdo }\end{array}$ \\
\hline Hablar de mis emociones & 35 & 5 & & & \\
\hline Expresar afecto & 25 & 15 & & & \\
\hline Confiar en las personas & 35 & 5 & & & \\
\hline $\begin{array}{l}\text { Superar dificultades que se me } \\
\text { presenten en la vida }\end{array}$ & 40 & & & & \\
\hline $\begin{array}{l}\text { Resolver problemas de manera } \\
\text { efectiva }\end{array}$ & 20 & 20 & & & \\
\hline Dar mi opinión & 10 & 10 & 10 & 10 & \\
\hline Buscar ayuda cuando la necesito & 20 & 5 & 15 & & \\
\hline Apoyar a otros que tienen dificultades & 40 & & & & \\
\hline Responsabilizarme por lo que hago & & 40 & & & \\
\hline Ser creativo & 30 & 5 & 5 & & \\
\hline Comunicarse adecuadamente & 35 & 5 & & & \\
\hline Aprender de mis aciertos y errores & 20 & 18 & 2 & & \\
\hline $\begin{array}{l}\text { Colaborar con otros para mejorar la } \\
\text { vida en la comunidad }\end{array}$ & 25 & 15 & & & \\
\hline Tomar decisiones & 15 & 15 & & 10 & \\
\hline $\begin{array}{l}\text { Generar estrategias para solucionar } \\
\text { mis problemas }\end{array}$ & 22 & 8 & 10 & & \\
\hline Fijarme metas realistas & & 40 & & & \\
\hline Esforzarme por lograr mis objetivos & & 35 & 5 & & \\
\hline Asumir riesgos & 10 & 20 & 10 & & \\
\hline Proyectarme al futuro & 40 & & & & \\
\hline
\end{tabular}

Tabla 9.- Factor de Resiliencia (Yo puedo)

Fuente: Descripción de los factores de Resiliencia (Yo puedo) del test SV-RES60. 


\section{Conclusiones}

Después de analizar los resultados arrojados por el grupo motivo de investigación se llegó a la siguiente conclusión.

Se demuestra que el deporte tiene características esenciales que permiten desarrollar de manera disciplinaria el carácter, la autoestima y la confianza en los federados de esta región. La resiliencia como factor internalizado está latente en cada uno de los mismos, desarrollando una metodología única en cada competir, como lo es el caso del deporte de ajedrez, que tienen la autoconfianza de regenerar la resiliencia de forma crítica para superar los problemas; además se aborda una dimensión inmediata en donde opera la resiliencia de forma adecuada a los deportistas motivo de investigación, los cuales dependen de las capacidades condicionales: velocidad, fuerza, resistencia y flexibilidad con hipervínculos de los proceso volitivo y cognoscitivos predominantes durante el proceso competitivo de los mismos para evaluar el nivel de resiliencia personal, adecuados en los deportes anteriormente mencionados. Permitiendo establecer una ventana abierta en el proceso de preparación de estos deportes, a través de la presencia del profesional en psicología de forma continúa para elevar la autoestima de los mismos. Los niveles de resilencia que presenta los deportistas de la federación de Manabí, cuentan con diferentes niveles de resiliencia, en esta caso, los ajedrecistas tienen mayor nivel de auto superación porque su deporte les exige la concentración y les permite tener control de las acciones que están emergidas en la autoconfianza, las disciplinas como boxeo y escalada, están expuestas a tendencias más baja ante la derrota, lo cual les impide una superación de adversidades, y e han logrado manifestar grados de resistencia y autovaloración más excedente ante los deportes.

\section{Bibliografías}

Álvarez, O, Estevan,I, Falcó, C, Castillo, I. (2013). Personas que estimulan mi autonomía e iniciativa. Personas que estimulan mi autonomía e iniciativa, 8 (1). 59-70.

APA. (2019). El camino hacia la Resiliencia. [ mensaje de un blog ]. Recuperado de https://www.apa.org/centrodeapoyo/resilienciacamino

Betron, S, Zurita, F, Cepero, M. (2016). LA RESILIENCIA COMO FACTOR
DETERMINANTE EN EL RENDIMIENTO DEPORTIVO. REVISIÓN BIBLIOGRÁFICA. e-balonmano.com: Revista de Ciencias del Deporte, 12 (2), 79-88. Recuperado de https://pdfs.semanticscholar.org/92f7/82c7961f 9079c825b13a368c43c071ae02a2.pdf

Cabanyes, J. (2010). Resiliencia una aproximación al concepto. Revista de psiquiatría y salud mental, 3 (4). 145-151. Recuperado de https://www.elsevier.es/es-revista-revistapsiquiatria-salud-mental-286-pdfS1888989110000741

Caldera, J, Aceves, B. (2016). Resiliencia en estudiantes universitarios. Un estudio comparado entre carreras. Psicogente, 19(36), 227-239.

http://doi.org/10.17081/psico.19.36.1294

Campos, G, Covarrubias, N, Martínez, E. (2012). La observación, un método para el estudio de la realidad. Xihmai, VII (13). 45-60. Recuperado de https://dialnet.unirioja.es $>$ descarga $>$ articulo

Chacón, R, Castro, M, Espejo, T, Zurita,F. (2016). Estudio de la resiliencia en función de la modalidad deportiva: fútbol, balonmano y esquí RETOS. Nuevas Tendencias en Educación Física, Deporte y Recreación, 29. 157-161. Recuperado de http://www.redalyc.org/pdf/3457/34574346403 2.pdf

Fandiño Parra, Yamith José (2011). Los jóvenes hoy: enfoques, problemáticas y retos. Revista Iberoamericana de Educación Superior, II(4). Recuperado de https://www.redalyc.org/articulo.oa?id=2991/2 99124247009

Finez, M, Moran, C. (2017). Resiliencia y autovaloraciones esenciales: estudio comparativo en adolescentes y jóvenes. Psychology, Society, \& Education, 9 (3). 347 356. doi: $10.25115 /$ psye.v9i3.857

García D (2016, noviembre 21). Definición y herramientas de la Resiliencia Organizacional. Recuperado de https://www.gestiopolis.com/definicionherramientas-la-resiliencia-organizacional/

Garcia del castillo, J, García del Castillo, A, López, C, Días, P. (2016). CONCEPTUALIZACIÓN TEÓRICA DE LA RESILIENCIA 
PSICOSOCIAL Y SU RELACIÓN CON LA SALUD. Salud y Drogas, 16 (1). 59-68. Recuperado

de https://www.redalyc.org/pdf/839/83943611006. pdf

Garcia, X, Molinero, O, Ruiz, R, Salguero, A, De la Vega, R, Márquez, S. (2014). La resiliencia en el deporte: fundamentos teóricos, instrumentos de evaluación y revisión de la literatura. Cuadernos de la Psicología del deporte, 14 (3). 83-98. Recuperado de https://www.researchgate.net/publication/2681 64838_La_resiliencia_en_el_deporte_fundame ntos_teoricos_instrumentos_de_evaluacion_y_r evision_de_la_literatura

Gómez, G, Rivas, M. (2017). RESILIENCIA ACADÉMICA, NUEVAS PERSPECTIVAS DE INTERPRETACIÓN DEL APRENDIZAJE EN CONTEXTOS DE VULNERABILIDAD SOCIAL. Calidad de la Educación, 47. 215-233. Recuperado de https://scielo.conicyt.cl/pdf/caledu/n47/07184565-caledu-47-00215.pdf

Oliva, E, Villa, V. (2013). Hacia un concepto interdisciplinario de la familia en la globalización. Justicia Juris, 10 (1). 11-20. Recuperado de http://www.scielo.org.co/pdf/jusju/v10n1/v10n 1a02.pdf

Ortega, Z, Mijares,B.(2018). CONCEPTO DE RESILIENCIA: DESDE LA DIFERENCIACIÓN DE OTROS CONSTRUCTOS, ESCUELAS Y ENFOQUES. Revista Científica Electrónica de Ciencias Humanas, 39 (13). 30-43. Recuperado de

http://www.revistaorbis.org.ve/pdf/39/art3.pdf

Peris, B. (2017). El deporte como fomento de resiliencia. ( tesis de grado). Universitat Jaume I. Recuperado de http://repositori.uji.es/xmlui/bitstream/handle/1 0234/169122/TFG_2017_PerisChiva_Berta.pdf ?sequence $=1$

Ramos, S. (2017). Niveles de resiliencia en una población afectada en un desastre natural. ( Tesis de pregrado). UDLA. Recuperado de de http://dspace.udla.edu.ec/bitstream/33000/7987 /1/UDLA-EC-TPC-2017-27.pdf
Sánchez, P, Gallardo, R, Ceña , F. (2016) La noción de resiliencia en el análisis de las dinámicas territoriales rurales: Una aproximación al concepto mediante un enfoque territorial. Cuadernos de Desarrollo Rural, 13(77), 93-116. http://dx.doi.org/10.11144/Javeriana.cdr1377.nrad

Tarazona, A, Saldarriaga, K, Vasquez, A, Pinargote, E, Dominguez, L. (CAPÍTULO 1. CUATRO ARISTAS DE LA RESILIENCIA EN LA PROVINCIA DE MANABÍ. ECUADOR). Recuperado de https://dialnet.unirioja.es/servlet/articulo?codig $\mathrm{o}=6805560$

Tomkiewicz, S. (2001). Resiliencia Individual. Capitulo de texto. Recuperado de https://www.avntf-evntf.com/wpcontent/uploads/2016/12/GomezB.Trab_.3BI09 10.pdf

Vidarte, J, Vélez, C, Sandoval, C, Alfonso,M. (2011). ACTIVIDAD FÍSICA: ESTRATEGIA DE PROMOCIÓN DE LA SALUD. Hacia la Promoción de la Salud, 16 (1). 202-219. Recuperado

de http://www.scielo.org.co/pdf/hpsal/v16n1/v16n 1a14.pdf. 